Delft University of Technology

\title{
Simulation of foam-assisted co2 storage in saline aquifers
}

Lyu, X.; Voskov, D.; Rossen, W.

DOI

10.3997/2214-4609.202035101

Publication date

2020

Document Version

Final published version

Published in

ECMOR 2020

\section{Citation (APA)}

Lyu, X., Voskov, D., \& Rossen, W. (2020). Simulation of foam-assisted co2 storage in saline aquifers. In ECMOR 2020 : 17th European Conference on the Mathematics of Oil Recovery (pp. 1-16). EAGE. https://doi.org/10.3997/2214-4609.202035101

\section{Important note}

To cite this publication, please use the final published version (if applicable).

Please check the document version above.

\section{Copyright}

Other than for strictly personal use, it is not permitted to download, forward or distribute the text or part of it, without the consent of the author(s) and/or copyright holder(s), unless the work is under an open content license such as Creative Commons.

\section{Takedown policy}

Please contact us and provide details if you believe this document breaches copyrights.

We will remove access to the work immediately and investigate your claim. 
Green Open Access added to TU Delft Institutional Repository

'You share, we take care!' - Taverne project

https://www.openaccess.nl/en/you-share-we-take-care

Otherwise as indicated in the copyright section: the publisher is the copyright holder of this work and the author uses the Dutch legislation to make this work public. 


\title{
Simulation of Foam-Assisted CO2 Storage in Saline Aquifers
}

\author{
X. Lyu ${ }^{1 *}$, D. Voskov ${ }^{1,2}$, W. Rossen ${ }^{1}$ \\ ${ }^{1}$ Delft University of Technology; ${ }^{2}$ Stanford University
}

\section{Summary}

Geological storage of $\mathrm{CO} 2$ is a crucial emerging technology to reduce anthropogenic greenhouse gas emissions. Due to the buoyant characteristic of injected gas and the complex geology of subsurface reservoirs, most injected $\mathrm{CO} 2$ either rapidly migrates to the top of the reservoir or fingers through high-permeability layers due to instability in the convection-dominated displacement. Both of these phenomena reduce the storage capacity of subsurface media. CO2-foam injection is a promising technology for reducing gas mobility and increasing trapping within the swept region in deep brine aquifers. A consistent thermodynamic model based on a combination of a classic cubic equation of state (EOS) for gas components with an activity model for the aqueous phase has been implemented to describe the phase behavior of the $\mathrm{CO} 2$-brine system with impurities. This phase-behavior module is combined with representation of foam by an implicit-texture (IT) model with two flow regimes. This combination can accurately capture the complicated dynamics of miscible $\mathrm{CO} 2$ foam at various stages of the sequestration process. The Operator-Based Linearization (OBL) approach is applied to reduce the nonlinearity of the $\mathrm{CO} 2$-foam problem by transforming the discretized conservation equations into space-dependent and statedependent operators. Surfactant-alternating-gas (SAG) injection is applied to overcome injectivity problems related to pressure build-up in the near-well region. In this study, a 3D large-scale heterogeneous reservoir is used to examine $\mathrm{CO} 2$-foam behaviour and its effects on $\mathrm{CO} 2$ storage. Simulation studies show foams can reduce gas mobility effectively by trapping gas bubbles and inhibit $\mathrm{CO} 2$ from migrating upward in the presence of gravity, which in turn improves remarkably the sweep efficiency and opens the unswept region for $\mathrm{CO} 2$ storage. We also study how surfactant injection and forming of foam affect enhanced dissolution of $\mathrm{CO} 2$ at various thermodynamic conditions. This work provides a possible strategy to develop robust and efficient CO2 storage technology. 


\section{Introduction}

Currently, due to various anthropogenic activities, the concentration of carbon dioxide $\left(\mathrm{CO}_{2}\right)$ in the atmosphere is having significant and observable effects on the environment. It's believed to be a major contributor to global climate change, such as rising sea level and ocean acidification (IPCC, 2014; NASA, 2018). Carbon capture, utilization and storage (CCUS) in subsurface geological formations have been proved to be one viable and promising solution for this environmental issue (Pruess et al., 2004; Raziperchikolaee et al., 2013; Alcorn et al., 2019; Ajoma et al., 2020). Deep saline aquifers are ideal sites for large scale long-term $\mathrm{CO}_{2}$ sequestration (Gale, 2004; Bachu et al., 2007; Li et al., 2018).

Since gas phases generally have higher mobility due to lower viscosity compared to the reservoir fluid, the injected $\mathrm{CO}_{2}$ will migrate along the top of the reservoir (Hesse et al., 2008). Along the progression, $\mathrm{CO}_{2}$ may leak into the atmosphere if it reaches faults or abandoned wells (Celia and Nordbotten, 2009). This effect also causes very poor sweep efficiency of $\mathrm{CO}_{2}$ (i.e., lowering storage capacity). These issues can be overcome or minimized by reducing gas mobility and increasing trapping within the pore space of the swept region. Simultaneous water and gas (SWAG) injection or water alternating gas (WAG) injection can improve $\mathrm{CO}_{2}$ sweep efficiency (Caudle et al., 1958; Bedrikovetsky, 2003). Laboratory studies have shown that SWAG and WAG injection reduce $\mathrm{CO}_{2}$ mobility and improve its sweep efficiency (Qi et al., 2009).

Foam injection is a promising technology for gas-mobility control in the petroleum industry and aquifer remediation (Rossen, 1996). Recently, the foam enhanced oil recovery (EOR) technique is being extended to $\mathrm{CO}_{2}$ storage, thus reducing greenhouse gas emissions (Vitoonkijvanich et al., 2015; Izadi and Kam, 2018; Alcorn et al., 2019; Dong et al., 2019). Foam is an agglomeration of gas bubbles separated from each other by thin liquid films, which can improve the sweep efficiency of injected gases by mitigating or reducing the effect of low gas viscosity and reservoir layers (Bikerman, 1973; Schramm, 1994; Rossen, 1996). Foam-assisted $\mathrm{CO}_{2}$ injection (i.e., adding surfactant to generate $\mathrm{CO}_{2}$ foams in situ) provides insights to maximize the potential of $\mathrm{CO}_{2}$ storage as well.

Fundamentally, capillary effects and the drag on foam films reduce gas mobility considerably (e.g. by $10 \sim 10^{4}$ times), through trapping gas bubbles (e.g. 90-99\% of gas) and increasing the flow resistance of flowing bubbles (Kil et al., 2011; Lyu et al., 2018). The reduction in gas mobility improves the sweep efficiency remarkably and opens otherwise unswept formation for $\mathrm{CO}_{2}$ storage. More $\mathrm{CO}_{2}$ thus is trapped in the pore space rather than migrate upward. The stress on the overburden rock is relaxed, reducing the risk of cracking it. As injection stops, nearly $100 \%$ of injected gas in the swept zone is trapped in-situ (as a discontinuous phase) by lamellae (Kil et al., 2011), as long as foam remains stable. The dispersion of $\mathrm{CO}_{2}$ in liquid increases the contact area of $\mathrm{CO}_{2}$ with rock and aqueous phase and thus improves storage capacity.

Prior to foam deployment, one needs to understand the following key issues. The first one is how to predict the behavior of the injected $\mathrm{CO}_{2}$ stream. In the post-injection period, the footprint of injected $\mathrm{CO}_{2}$ plays an important role in the security and permanence of $\mathrm{CO}_{2}$ storage (Li et al., 2018). The second important phenomenon is the residual trapping of $\mathrm{CO}_{2}$ during the migration through the saline aquifer; then dissolution starts to play a significant role at longer timescales. We need an accurate model to represent the major physical and chemical processes induced by $\mathrm{CO}_{2}$ foam injection into potential disposal reservoirs. Last, but not least, the nonlinearity of this coupled process challenges conventional simulation, which often translates into an extreme computational cost. It is essential to establish a robust and accurate simulation technique which can model these processes in a realistic and quantitative fashion (Pruess et al., 2004).

In this work, therefore, we study the coupling of $\mathrm{CO}_{2}$ sequestration with foam injection. For an accurate description of the phase behavior, a recently developed thermodynamic model based on a combination of cubic Equation of State (EOS) with an activity model has been implemented (Ziabakhsh-Ganji and Kooi, 2012). This model combines a classic fugacity-based formulation for the supercritical gas phase and an activity model combined with Henry's constants for the aqueous brine. This implementation makes the thermodynamic model more accurate than conventional cubic EOS. The implicit-texture (IT) model (CMG-STARS, 2012) is used in this study, assumes that foam generation and destruction reach a local steady-state instantaneously and represents the effect of foam bubbles implicitly by introducing a mobility-reduction factor. This mobility-reduction factor, used to rescale gas mobility with foam, is a function of water saturation, oil saturation, surfactant concentration, capillary number and salinity. 
To accurately simulate these highly nonlinear coupled foam-assisted $\mathrm{CO}_{2}$ storage processes, a new approach, named Operator-Based Linearization (OBL), where performance, flexibility and robustness can be combined without compromise, is introduced to reduce the nonlinearity of complex physical problems (Khait and Voskov, 2018). The OBL approach transforms the discretized mass-conservation equations to space-dependent and state-dependent operators. While space-dependent operators are treated conventionally, the state-dependent operators are approximated by discrete representation on a uniform mesh in parameter-space. These state-dependent operators rely on current local physical properties (e.g. density, viscosity, relative permeability), which represent the most nonlinear part of the governing equations. The continuous representation of these operators is achieved through the multilinear interpolation, which provides a unique tool for approximate representation of the exact physics of the problem. The OBL approach also provides an opportunity to control the nonlinearity in physics by changing the resolution of parameter space.

This paper is structured as follows. First, we briefly describe our numerical and thermodynamic models. Then we validate our simulation capabilities against analytical solutions, mainly focusing on the enhanced $\mathrm{CO}_{2}$ dissolution. Furthermore, we investigate the behavior of the $\mathrm{CO}_{2}$ plume with brine-assisted (co-injecting $\mathrm{CO}_{2}$ and brine) and foam-assisted (co-injecting $\mathrm{CO}_{2}$ and surfactant solution) $\mathrm{CO}_{2}$ injection, including the plume footprint, the amount of $\mathrm{CO}_{2}$ dissolved and residually trapped, storage capacity and efficiency using an unstructured 3D reservoir with homogeneous properties. We conclude the paper by summarizing the main conclusions.

\section{Methodology}

\section{Governing equations}

In this section, we briefly consider the governing equations and nonlinear formulation for two-phase multi-component isothermal flow in porous media:

$$
\frac{\partial}{\partial t}\left(\phi \sum_{j=1}^{n_{p}} x_{c j} \rho_{j} s_{j}\right)+\operatorname{div} \sum_{j=1}^{n_{p}}\left(x_{c j} \rho_{j} \mathbf{u}_{j}+s_{j} \rho_{j} \mathbf{J}_{c j}\right)+\sum_{j=1}^{n_{j}} x_{c j} \rho_{j} \widetilde{q}_{j}=0, \quad c=1,2 \cdots, n_{c}
$$

where subscript $j \in\{w, n\}$ denotes the wetting phase (brine) and the nonwetting phase (supercritical $\mathrm{CO}_{2}$ ). $\phi$ is porosity, $s_{j}$ is phase saturation, $\rho_{j}$ is phase molar density, $x_{c j}$ is component mole fraction in a phase. $\mathbf{u}$ is Darcy velocity, $\mathbf{J}$ is Fick's diffusion flux.

In addition, Darcy's law is applied to describe the flow of each phase:

$$
\mathbf{u}_{j}=-k \frac{k_{r j}}{\mu_{j}}\left(\nabla p_{j}-\rho_{j} g \nabla D\right)
$$

where $k$ is permeability tensor, $k_{r j}$ is relative permeability, $\mu_{j}$ is phase viscosity, $p_{j}$ is pressure in phase $j, g$ is the vector of gravitational acceleration. $D$ is the depth.

In the presence of capillarity, $p_{j}$ is different between wetting phase and non-wetting phase:

$$
p_{w}=p_{n}-p_{c}
$$

where $p_{c}$ is capillary pressure, which relates the pressures of the two phases. $p_{n}$ is the non-wetting phase, $p_{w}$ is the wetting phase. Capillary pressure is a function of saturation, often expressed as $p_{c}\left(s_{w}\right)$. The capillary-pressure-saturation relationship, also called the capillary-pressure curve, can be measured in laboratories.

Generally, nearly all foam models alter only the transport properties of gas and assume that liquid properties remain the same function of saturations as in the absence of foam, which is in accordance with laboratory investigations (Friedmann et al., 1991; Rossen, 1996; Dholkawala et al., 2007; Lotfollahi et al., 2016). In the presence of foam, gas is trapped by stationary lemallae to reduce gas mobility. In the implicit-texture foam model (CMG-STARS, 2012) studied here, foam reduces gas mobility by modifying gas relative permeability with a mobility-reduction factor $(F M)$ as shown below:

$$
k_{r g}^{f}=k_{r g} \times F M
$$




$$
F M=\frac{1}{1+f m m o b \times F_{1} \times F_{2} \times F_{3} \times F_{4} \times F_{5} \times F_{6}}
$$

where $k_{r g}^{f}$ and $k_{r g}$ are gas relative permeability with and without foam, respectively; fmmob is defined as the maximum-attainable gas-mobility reduction, and $F_{1}$ through $F_{6}$ are functions accounting for the effects of physical factors on gas mobility (e.g., surfactant concentration, water saturation, oil saturation, oil composition, capillary number, and salinity). In this project, we consider only two functions, $F_{1}$ and $F_{5}$, capturing the effects of surfactant concentration and water saturation on foam strength. The details are found in Rossen et al. (2010).

$\mathbf{J}_{c j}$ is the diffusion-dispersion tensor of component $c$ in phase $j$, which is described by

$$
\mathbf{J}_{c j}=-\phi D_{c j} \nabla x_{c j}
$$

where $x$ is mass fraction, $D$ is diffusion coefficient.

The saturation constraint requires

$$
\sum_{j=1}^{n_{p}} s_{j}=1
$$

where $s_{j}=\frac{v_{j} / \rho_{j}}{\sum_{j=1}^{n_{j}} v_{j} / \rho_{j}}$ and $v_{j}$ is the molar fraction of phase $j$.

A finite-volume discretization on a general structured mesh and backward Euler approximation in time is applied:

$$
V\left(\left(\phi \sum_{j=1}^{n_{p}} x_{c j} \rho_{j} s_{j}\right)^{n+1}-\left(\phi \sum_{j=1}^{n_{p}} x_{c j} \rho_{j} s_{j}\right)^{n}\right)-\Delta t \sum_{l}\left(\sum_{j=1}^{n_{p}}\left(x_{c j}^{l} \rho_{j}^{l} \Gamma_{j}^{l} \psi^{l}+s_{j}^{l} \rho_{j}^{l} D_{c j}^{l} \Gamma_{f}^{l} \Delta x_{c j}^{l}\right)\right)+\Delta t \sum_{j=1}^{n_{p}} x_{c j} \rho_{j} q_{j}=0
$$

where $\Gamma_{j}^{l}=\Gamma^{l} k_{r j}^{l} / \mu_{j}^{l}$ is a phase $j$ transmissibility over interface $l$, and $\Gamma^{l}$ is the constant geometrical part of transmissibility. $\Gamma_{f}^{l}$ is the transmissibility of diffusion term, including the geometry of the control volumes connected by interface $l$ and rock porosity. $\psi^{l}$ is the pressure difference between neighbor cells. Eqs. 1 is approximated in space using a two-point flux approximation (TPFA) and in time using a fully implicit method. This introduces strong nonlinearity into the system of the governing equations, especially in the presence of complicated physics. In our simulation, we use the overall composition of the mixtures and pressure as the primary unknown variables. Then we need to linearize the problem, which requires determining all the partial derivatives with respect to these nonlinear unknowns and assembling the Jacobian and residuals. After the linearization step, the Newton-Raphson method is adopted to solve the linearized system of equations on each nonlinear iteration in the following form:

$$
J\left(\omega^{k}\right)\left(\omega^{k+1}-\omega^{k}\right)=-r\left(\omega^{k}\right)
$$

where $J\left(\omega^{k}\right)$ and $r\left(\omega^{k}\right)$ are the Jacobian and residual defined at the nonlinear iteration $k$, and $\omega$ is the primary unknown variables. In conventional simulation, the Jacobian should be assembled with accurate numerical property values and their derivatives with respect to nonlinear unknowns. This process requires either various interpolations (for properties such as relative permeabilities of different phases) or solution of a highly nonlinear system in combination with the chain rule and inverse theorem, which could increase the computational cost.

\section{OBL approach}

Following the OBL approach, all variables in the Eqs.1, are expressed as a product of state-dependent $(\omega)$ and space-dependent $(\xi)$ operators (Khait and Voskov, 2017). The discretized mass-conservation equation in operator form is

$$
V \phi_{0}\left[\alpha_{c}(\omega)-\alpha_{c}\left(\omega_{n}\right)\right]+\Delta t \sum_{l \in L(i)}\left(\Gamma^{l} \Psi^{l} \beta_{c}(\omega)+\Gamma_{f}^{l} \Delta \gamma(\omega)^{l}\right)+\theta(\xi, \omega, u)=0
$$


Here,

$$
\begin{aligned}
\alpha_{c}(\omega) & =\left(1+c_{r}\left(p-p_{r e f}\right)\right) \sum_{j=1}^{n_{p}} x_{c j} \rho_{j} s_{j} \\
\beta_{c}(\omega) & =\sum_{j=1}^{n_{p}} x_{c j} \rho_{j} k_{r j} / \mu_{j} \\
\gamma(\omega) & =\sum_{j=1}^{n_{p}} \rho_{j} s_{j} D_{c j} x_{c j} \\
\theta(\xi, \omega, u) & =\Delta t \sum_{j=1}^{n_{p}} x_{c j} \rho_{j} q_{j}(\xi, \omega, u)
\end{aligned}
$$

where $\omega$ and $\omega_{n}$ are nonlinear unknowns in the current and previous timestep, respectively; $L(i)$ is the set of neighbors of the control volume $l ; \theta(\xi, \omega, u)$ is the source term. $V, \phi_{0}$, and $c_{r}$ are initial volume, porosity and rock compressibility, respectively. $\rho_{p}, k_{r p}$, and $\mu_{p}$ are phase density, phase relative permeability and phase viscosity, respectively. In our problem, both effects of gravity and capillarity are considered, which means the phase potential differences are not equal across all phases at any interface. In the presence of foam, the gas flux operator should be rescaled by the mobility reduction factor.

This representation allows us to decouple a computation of nonlinear physics from conventional discretization terms. More details can be found in Voskov (2017) and Khait and Voskov (2018).

\section{Thermodynamic model}

The model describes thermodynamic equilibrium between a non-aqueous phase (i.e., a multi-component mixture which can be in gas, supercritical or condensed conditions) and an aqueous phase (i.e., liquid which includes dissolved hydrocarbon and gases). Due to the instantaneous local equilibrium assumption, phase-behaviour calculations are decoupled from flow and transport. In a multi-phase system, an exact thermodynamic equilibrium is required at every nonlinear iteration in the molar formulation

$$
\begin{aligned}
z_{c}-\sum_{j=1}^{n_{p}} v_{j} x_{c j} & =0 \\
f_{c 1}\left(p, T, x_{1}\right)-f_{c j}\left(p, T, x_{j}\right) & =0 \\
\sum_{c=1}^{n_{c}}\left(x_{c 1}-x_{c j}\right) & =0 \\
\sum_{j=1}^{n_{p}} v_{j}-1 & =0
\end{aligned}
$$

Here $z_{c}=\sum_{j} x_{c j} \rho_{j} s_{j} / \sum_{j} \rho_{j} s_{j}$ is overall composition and $f_{c j}\left(p, T, x_{c j}\right)$ is the fugacity of component $c$ in phase $p$. The set of thermodynamic relations described by Eq. 15 to Eq. 18 must be simultaneously solved for the conditions of pressure, temperature and composition in each grid block in the nonlinear loop.

In this work, a fugacity-activity model is used to solve for thermodynamic equilibrium based on the idea originally proposed by Kritchevsky and Iliinskaya (1945). In this approach, the fugacity of the gas phase is expressed in terms of the fugacity coefficient and the aqueous phase in terms of activity:

$$
\begin{gathered}
f_{i}^{g}=p \Phi_{i} y_{i} \\
f_{i}^{l}=h_{i} \gamma_{i} x_{i}
\end{gathered}
$$

where $p$ is the total pressure in the system, $\Phi$ the fugacity coefficient of the gas phase, $h_{i}$ Henry's constant, $\gamma_{i}$ activity coefficient, $x_{i}$ and $y_{i}$ the molar fraction of each component in aqueous phase and gas phase, respectively. 
Insertion of the expressions for the vapour and aqueous phase fugacities gives the following expression of the thermodynamic equilibrium:

$$
p \Phi_{i} y_{i}=h_{i} \gamma_{i} x_{i}
$$

The above equation can be rearranged to obtain the phase-equilibrium constant of each component $K_{i}$ :

$$
K_{i}=\frac{y_{i}}{x_{i}}=\frac{h_{i} \gamma_{i}}{p \Phi_{i}}
$$

Eq. 22 is used to calculate $K$ values for different gas components. The equilibrium constant for the water component is calculated with a separate relation proposed by Spycher et al. (2003):

$$
K_{\mathrm{H}_{2} \mathrm{O}}=\frac{y_{\mathrm{H}_{2} \mathrm{O}}}{x_{\mathrm{H}_{2} \mathrm{O}}}=\frac{K_{\mathrm{H}_{2} \mathrm{O}}^{0}}{\Phi_{\mathrm{H}_{2} \mathrm{O}}} \exp \left(\frac{(p-1) V_{\mathrm{H}_{2} \mathrm{O}}}{R T}\right)
$$

where $K_{\mathrm{H}_{2} \mathrm{O}}^{0}$ is the equilibrium constant of $\mathrm{H}_{2} \mathrm{O}$ at the reference pressure of $1 \mathrm{bar}, T$ is temperature in Kelvins, $V_{\mathrm{H}_{2} \mathrm{O}}$ molar volume of $\mathrm{H}_{2} \mathrm{O}$. More detailed description can be found in Spycher et al. (2003).

Phase calculations are performed on all phases and phase partitioning is calculated using negative flash as described by Iranshahr et al. (2010) with successive substitution iteration. In order to initiate the negative-flash procedure, composition-independent ideal K-values provide an initial guess of phase fractions. Then, based on the output of the first iteration (phase fractions and composition of each phase), fugacity coefficients are updated to obtain new K-values. Once the thermodynamic system is solved, the thermophysical properties associated with the mass-conservation equations, such as phase density and phase viscosity, can be determined. The accuracy of this thermodynamic model vs. experimental results has been validated in Morshuis (2019).

Foam-assisted $\mathrm{CO}_{2}$ storage simulations for a brine aquifer are performed with the Delft Advanced Research Terra Simulator (DARTS) which is capable of modeling complex flow and transport related to various energy applications (Khait and Voskov, 2017; Kala and Voskov, 2020; Wang et al., 2020).

\section{Enhanced dissolution}

We begin by validating our simulation approach through studying the detailed behavior of gravity induced instabilities and the associated dissolution rate in small domains. Elenius et al. $(2012,2014)$ investigated the full problem of two-phase flow with gravity currents and convective dissolution in the absence and presence of the capillary transition zone (CTZ), and these results can be used as a benchmark for verification of our simulation approach. In this work, we take two small models, as shown in Fig. 1. One represents a scenario where the CTZ is negligible, and another one is with a realistic capillary transition zone. All the parameters which are used in the simulations and the simplifications in these models can be found in Elenius et al. (2015).

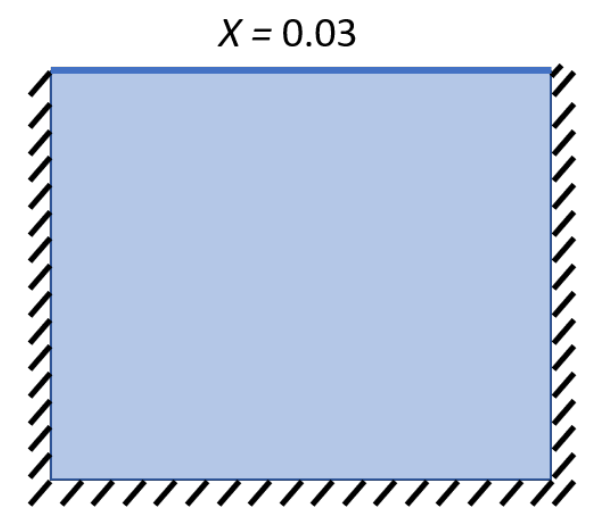

(a) Singe-phase flow

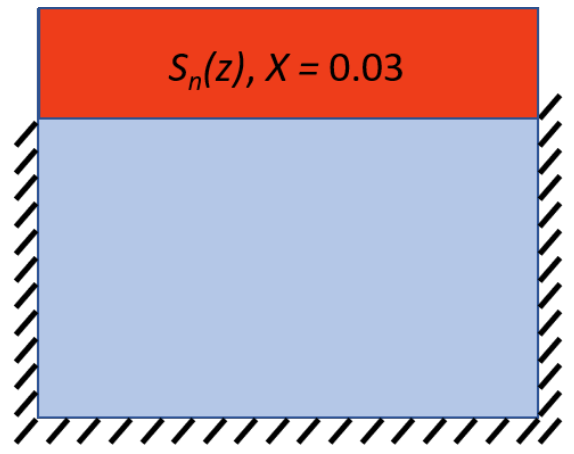

(b) a static CTZ (by high pore volume)

Figure 1: Schematic model used in this study. Initial position of region with brine (blue, $\mathrm{X}=0 \mathrm{~kg} / \mathrm{kg}$ ) and two-phase conditions (red, $\mathrm{X}=0.03 \mathrm{~kg} / \mathrm{kg}$ ). 
Fig. 2 demonstrates the $\mathrm{CO}_{2}$ concentration for the simulation after 200 years with single-phase brine and the simulation with a stagnant CTZ. Obviously, the stagnant CTZ enhances the concentration of $\mathrm{CO}_{2}$ in the fingers away from the interface, leading to a faster propagation of the fingers, compared with the no-flux top boundary case. This is consistent with the findings of Elenius et al. $(2012,2014)$. Therefore, we can infer that the presence of the stagnant CTZ, to some extent, can improve the storage efficiency by enhancing dissolution rate.

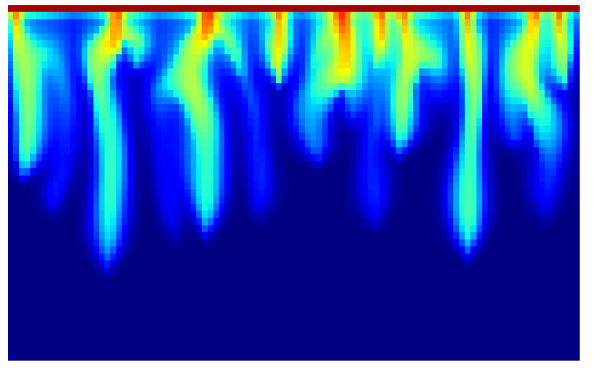

(a) Singe-phase flow

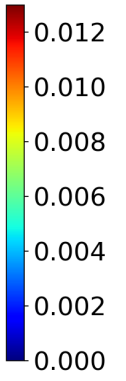

000

Figure 2: Fingers of dissolved $\mathrm{CO}_{2}$ concentration $(\mathrm{mol} / \mathrm{mol})$ at 200 years for the simulations.

Following the definition of dissolution rate in Elenius et al. (2015), we calculate the rate of $\mathrm{CO}_{2}$ mass transfer to the (single-phase) brine region across the interface per area (length) of the top interface:

$$
F=h \phi \frac{\partial \bar{c}}{\partial t}
$$

where $h$ and $\bar{c}$ are the thickness and mean concentration of the single-phase brine region respectively.

Elenius et al. (2014) also provided a semi-analytical solution for the dissolution rate with the effect of the capillary transition zone:

$$
F=(-0.011 \log (d)+0.016) \frac{K \Delta \rho_{w} g X_{\max } \rho_{w}\left(X_{\max }\right)}{\mu_{w}}
$$

and at negligible effect of the transition zone:

$$
F=0.021 \frac{K \Delta \rho_{w} g X_{\max } \rho_{w}\left(X_{\max }\right)}{\mu_{w}}
$$

where $K$ is permeability, $\Delta \rho_{w}$ density difference between brine and brine with dissolved $\mathrm{CO}_{2}, g$ gravitational acceleration, $X_{\max }$ maximum solubility, $\mu_{w}$ water viscosity, and $d$ the exponent of the relativepermeability function which is obtained by fitting the water relative permeability.

Fig. 3 displays the comparisons between the dissolution rates obtained in simulations (single-phase and $\mathrm{CTZ}$ ) and by the analytical equation. At the early time, diffusion dominates the mass transfer and the dissolution rate is reduced with time until the nonlinear onset time is reached. It also shows that the presence of CTZ can reduce the onset time. After the nonlinear onset time, fingers start growing and the rate increases due to convection. For the single-phase simulation, the dissolution rate stabilizes close to the analytical solution.

As shown in Fig. 3, the dissolution is reduced at late times when $\mathrm{CO}_{2}$ fingers approach the bottom of the aquifer. Here we use the stagnant $\mathrm{CTZ}$ to investigate the behavior of fingers at late times. $\mathrm{CO}_{2}$ starts to dissolve in brine and fill up the domain gradually (Fig. 4(a)). But the dissolution rate is reduced at late time mainly because of the merging of fingers and the increase of overall $\mathrm{CO}_{2}$ concentration. After 3000 years, $\mathrm{CO}_{2}$ concentration is already rather high, though it is still below the solubility limit anywhere in the single-phase brine region. Our simulation results with the CTZ show a similar $t_{\text {peel }}=$ 350 years, i.g., the time at which the dissolution rate starts to decrease, which is consistent with Slim (2014)'s findings. After $t_{\text {peel }}$, Slim also found the dissolution decreases from a constant value to a value proportional to $1 /(t+g)^{2}$ without a CTZ $\left(1 / t^{2}\right.$ in Elenius et al., 2015). Here we fit the coefficient $g$ based on our simulation results with the CTZ, and $g \approx-1000$ gives a good match (Fig. 4(b)). These results validate the greater accuracy of our enhanced dissolution model. 


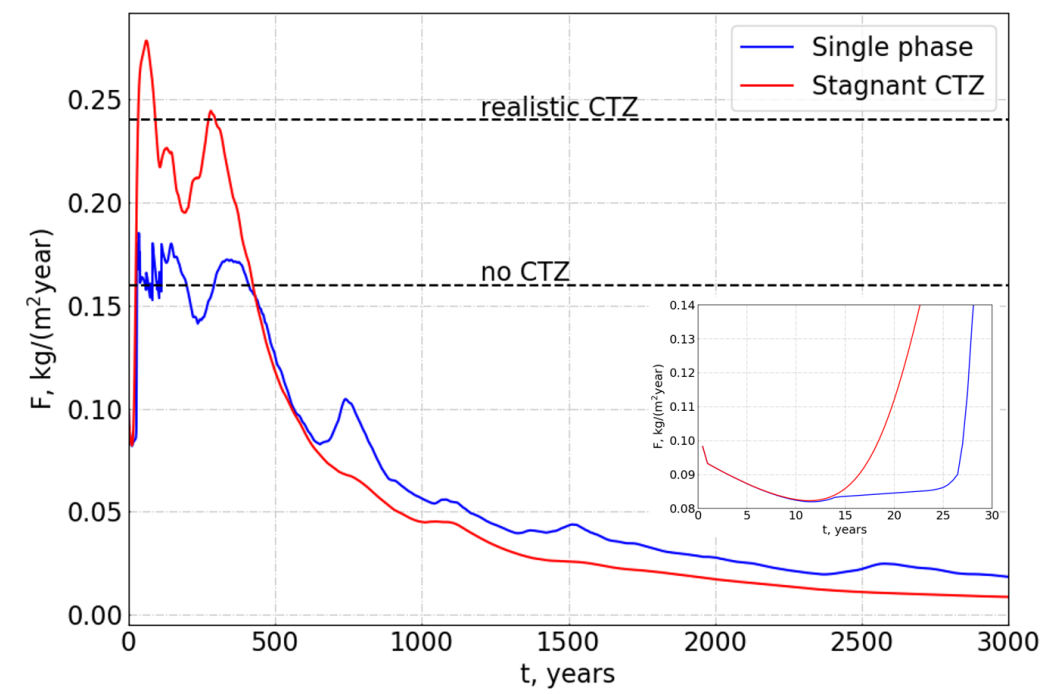

Figure 3: Mass flux of $\mathrm{CO}_{2}$ into the single-phase brine region. The black dashed lines are reported rates obtained by Eq. 25 and Eq. 26 in the presence and absence of the CTZ. The subfigure inside shows the mass flux at early times.

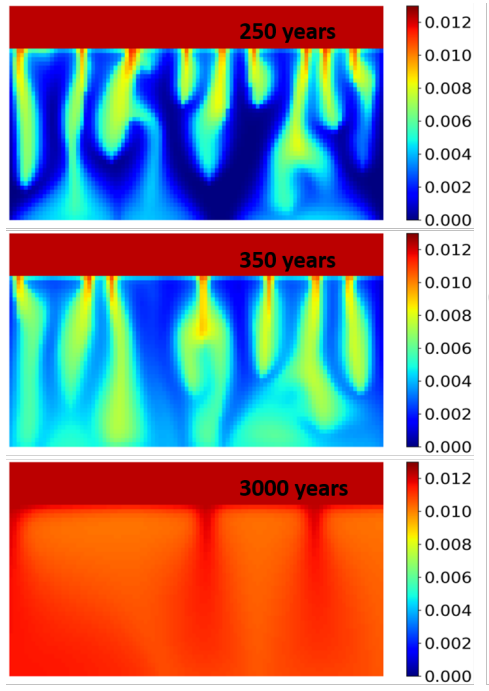

(a)

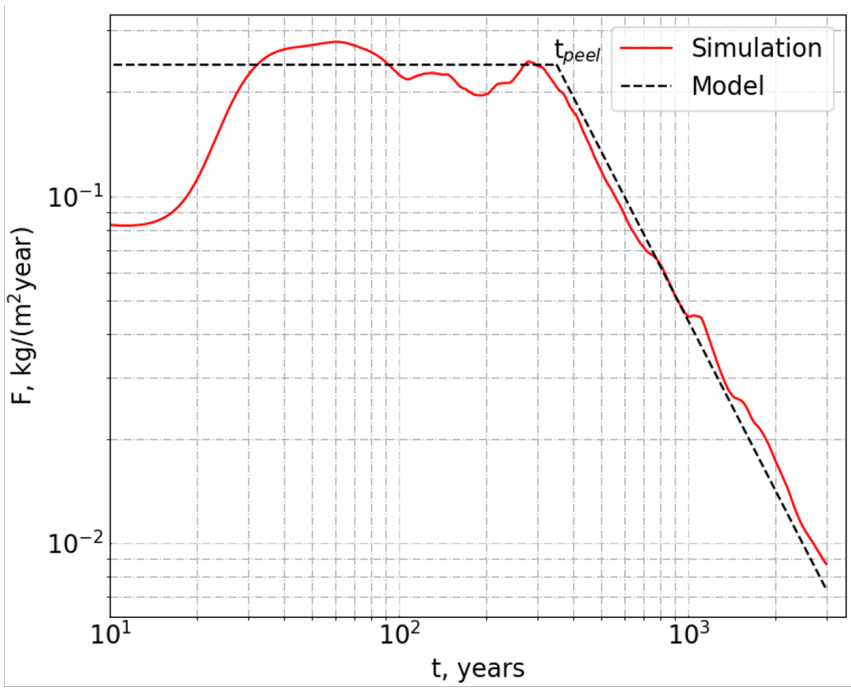

(b)

Figure 4: Late-time dissolution with a stagnant CTZ. (a) $\mathrm{CO}_{2}$ molar concentration (mol $/ \mathrm{mol}$ ) at different time, and (b) dissolution rate.

\section{Simulation of foam-assisted $\mathrm{CO}_{2}$ storage}

\section{Model description}

When $\mathrm{CO}_{2}$ is injected into a realistic formation saturated with brine, it migrates upwards due to gravity and forms a nearly horizontal layer overlying the brine phase. After a short time, $\mathrm{CO}_{2}$ starts to dissolve in the brine resulting from molecular diffusion and density-driven convection flow and in part is trapped in situ as residual gas. Many researchers have found that foam-assisted $\mathrm{CO}_{2}$ injection can increase sweep efficiency by mitigating gravity segregation processes (Vitoonkijvanich et al., 2015; Izadi and Kam, 2018). Therefore, it can increase the storage capacity due to the larger swept area and the increasing residual gas saturation.

In order to simulate this process, we consider a 3D homogeneous horizontal reservoir with unstructured mesh and fine mesh size as shown in Fig. 5. The height and the radius of the model is $30 \mathrm{~m}$ and $400 \mathrm{~m}$, 
respectively. There are 30 layers and the average number of elements in radial direction is 192 . Other parameters, such as rock and fluid properties, are listed in Table.1. Although the scale of this model is just a few hundred meters, it provides an accurate representation of $\mathrm{CO}_{2}$ sequestration with realistic thermodynamics conditions. As shown in Elenius et al. (2015), the proposed mesh resolution (around meter scale) provides a numerically converged solution for enhanced dissolution phenomena, which is studied here in a fully 3D setting.

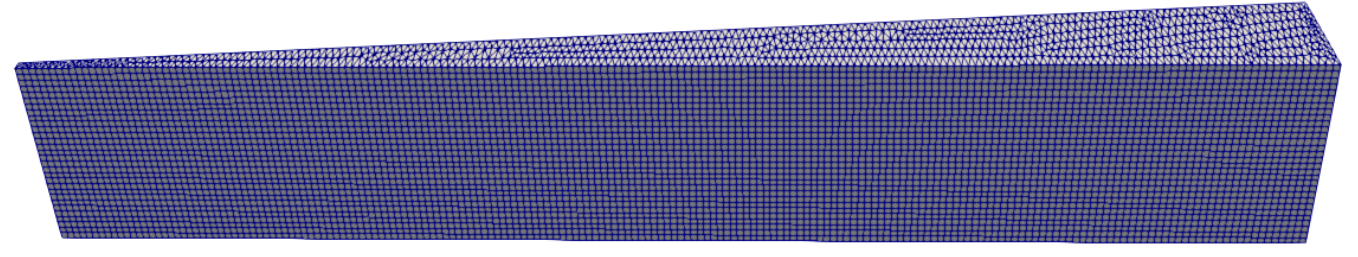

Figure 5: Schematic representation of the geometry model used in this study.

Table 1: Input parameters for the three-dimensional model

\begin{tabular}{lcll}
\hline \multicolumn{4}{c}{ Reservoir Properties } \\
\hline Average mesh size, $\mathrm{m}^{3}$ & 1.556 & Total number of elements & 49320 \\
Permeability, md & 100 & Porosity & 0.3 \\
Initial water saturation & 1.0 & Initial temperature, ${ }^{o} \mathrm{C}$ & 50 \\
Capillary entry pressure, bar & 0.2 & Initial pressure, bar & 90 \\
Corey gas exponent & 2.0 & Corey water exponent & 4.0 \\
Residual gas saturation & 0.2 & Connate water saturation & 0.2 \\
Endpoint gas relative permeability & 0.4 & Endpoint relative permeability & 1.0 \\
Diffusion coefficient, $\mathrm{m}^{2} /$ day & $2 \times 10^{-5}$ & \\
\hline \multicolumn{5}{c}{ Injection condition } \\
\hline Gas injection rate, $\mathrm{rm}^{3} /$ day & 4.0 & Water injection rate, $\mathrm{rm}^{3} /$ day & 1.0 \\
\hline \multicolumn{5}{c}{ Foam parameters } \\
\hline fmdry & 0.35 & epdry & 1000 \\
fmmob & 100 & & \\
\hline
\end{tabular}

To simplify the problem, we neglect any chemical reactions imposed in the brine by interactions with $\mathrm{CO}_{2}$ phase, such as $\mathrm{CO}_{2}$-rock mineral reactions and $\mathrm{CO}_{2}$-brine dissociation. Besides, the temperature assumed to be constant during the simulation. The simulation domain, a $5^{\circ}$ sector of the cylinder, is initially saturated with formation brine with no dissolved $\mathrm{CO}_{2}$. The injection well fully perforating the entire vertical interval is located at the left boundary and constant pressure is assumed at right boundary with no-flow conditions along the rest of interfaces. A fixed gas injection rate of $4.0 \mathrm{~m}^{3} / \mathrm{day}$, corresponds to $0.06 \mathrm{Mt} /$ year normalized to $360^{\circ}$. The injection well is closed after one year of injection.

Another simplification is the model of gas trapping due to the presence of foam. Gas trapping is an important mechanism in the foam-assisted $\mathrm{CO}_{2}$ storage process, especially after injection. Friedmann et al. (1991) measured trapped gas fractions in the range $75 \%$ to $90 \%$ over a wide range of velocities. Tang and Kovscek (2006) found a significant decrease in trapped gas with increasing gas velocity. Jones et al. (2018) also found in micro-models that as the superficial velocity increases, the fraction of trapping gas decreases. There is no complete models to describe the amount of trapped gas due to the injection of foam. In our study, for simplicity, we assume the residual (i.e., trapped) gas saturation rises by 0.1 in the presence of foam. This assumption is not rigorously correct because, as noted, the trapped gas saturation with foam is larger. In addition, in the upper layer where foam is collapsed or can't be generated, the residual saturation doesn't change. During the simulation, only one set of relative permeability curve is used. However, gas saturation is much larger than $S_{g r}$ and the only effect of this assumption is a modest reduction in $k_{r g}$. 


\section{Results and discussion}

In this section, we present the results of brine-assisted and foam-assisted $\mathrm{CO}_{2}$ injection into a homogeneous reservoir, including the behavior of the $\mathrm{CO}_{2}$ plume in injection and subsequent post-injection processes.

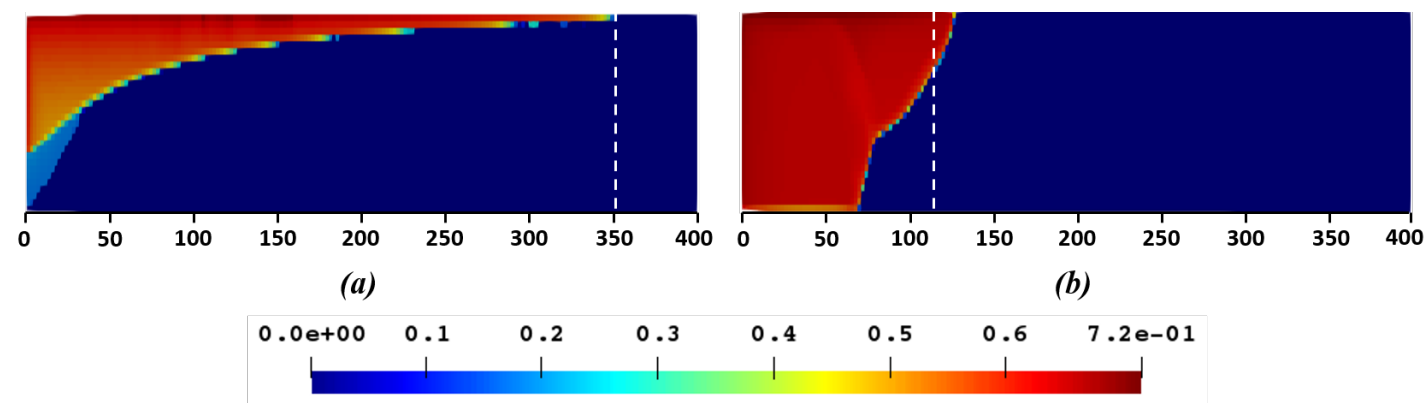

Figure 6: Saturation of supercritical $\mathrm{CO}_{2}$ after 1 year injection. (a) brine-assisted; (b) foam-assisted. The white dash line is the $\mathrm{CO}_{2}$ front.

The injected $\mathrm{CO}_{2}$ exists as supercritical fluid under the selected reservoir conditions. Fig. 6 illustrates the saturation of the supercritical $\mathrm{CO}_{2}$ after 1 year injection. During the brine-assisted $\mathrm{CO}_{2}$ injection, gas segregates with water and migrates upwards quickly because of the low density and viscosity of $\mathrm{CO}_{2}$ compared with the formation brine. In the meantime, it displaces the formation brine and thereby increases the contact area for $\mathrm{CO}_{2}$ storage. The plume, however, sweeps only the near-well region and then rises to the upper layer. Thus the storage efficiency, especially in the near-well region, is rather low due to the limited swept region.

Foam injection can significantly enlarge the swept area by reducing gas mobility. When $\mathrm{CO}_{2}$ and surfactant are co-injected into the formation, foam can be generated in the near-well region; then gas mobility is reduced remarkably (max. 100 times in this study) and much more space will be open for $\mathrm{CO}_{2}$ storage (Fig. 6(b)). The plume front in foam injection moves slowly and uniformly, which reduces the risk of leakage, especially during $\mathrm{CO}_{2}$ EOR processes where wells distance is limited.

Under steady-state, an analytical model for uniform co-injection of water and gas in homogeneous, horizontal reservoirs can be used to predict the segregation length (Stone et al., 1982). In this study, only 0.06 Pore Volume (PV) of gas is injected. No obviously separated regions, therefore, can be distinguished with a sharp boundary compared with the previous research (Stone et al., 1982; Rossen et al., 2010). However, in this transient displacement process, foam exhibits its capacity to hinder gas rising upwards and increase the sweep area. Fig. 6(b) shows that the segregation point where water and gas separate completely, is around $100 \mathrm{~m}$ from the injection well. There is an override zone forming in the upper layer beyond the segregation point. The similar observations can be found in Rossen et al. (2010) for $\mathrm{CO}_{2}$ injection for EOR. At early time, foam may reduce the dissolution rate due to the reduced contact area between $\mathrm{CO}_{2}$ and brine in the upper layers. However, in the long run, the dissolution increases because the free gas after segregation as well as collapsed foam still migrates upwards to overlie the brine phase in the upper layer, thus increasing the contact area.

Fig. 7 and Fig. 8 display the saturation of the supercritical $\mathrm{CO}_{2}$ with time. In both cases, the mobile $\mathrm{CO}_{2}$ forms a nearly horizontal layer overlying the brine phase. As shown in Fig. 6(a), when injection ceases, the front of $\mathrm{CO}_{2}$ plume approaches the right boundary. Therefore, the $\mathrm{CO}_{2}$ plume arrives at the right boundary in a short time in the post-injection process. With the dissolution of $\mathrm{CO}_{2}$ in the upper part of reservoir, the leading tip retracts and disappears gradually (Fig. 7(a) and Fig. 8(a)). After foam injection, gravitational force dominates the flow, and gas migrates upwards and accumulates there. Once gas saturation is high enough (i.e., water saturation is lower than the limiting water saturation) in the upper layer, foam collapses and gas mobility increases dramatically. Foam cannot be re-generated there, which makes the override zone thin in the foam-assisted post-injection process (Fig. 7(b) and Fig. 8(b)). Foam-injection retards the late-time dissolution rate. However, the residual trapped $\mathrm{CO}_{2}$ phase with foam-assisted injection is much greater than that of brine-assisted injection, in terms of the swept area and saturation of immobile gas. Foam increases the swept area and during the post-injection 

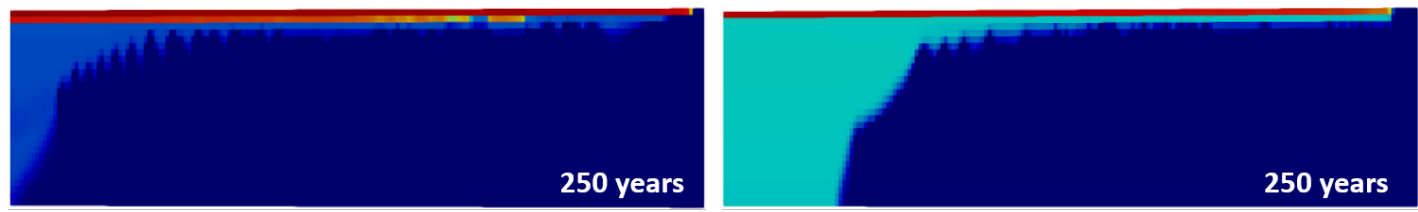

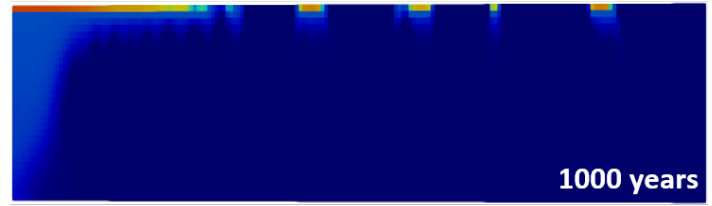

(a)



(b)

$\begin{array}{lllllllll}0.0 e+00 & 0.1 & 0.2 & 0.3 & 0.4 & 0.5 & 0.6 & 0.7 & 8.0 \mathrm{e}-01\end{array}$

Figure 7: Saturation of supercritical $\mathrm{CO}_{2}$ (front view) after 250 and 1000 years. (a) brine-assisted; (b) foam-assisted.

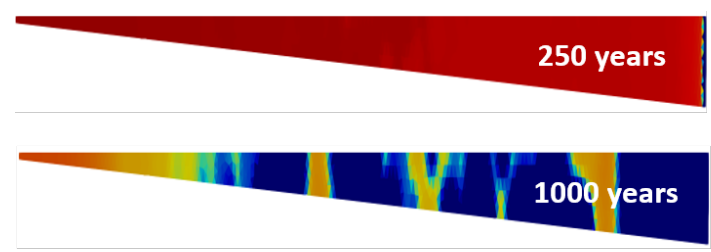

(a)

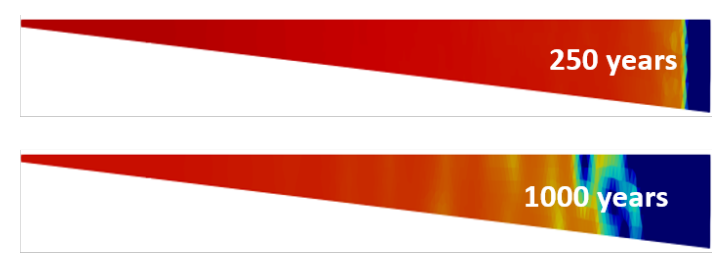

(b)

$\begin{array}{ccccccccc}0.0 e+00 & 0.1 & 0.2 & 0.3 & 0.4 & 0.5 & 0.6 & 0.7 & 8.0 e-01\end{array}$

Figure 8: Saturation of supercritical $\mathrm{CO}_{2}$ (top view) after 250 and 1000 years. (a) brine-assisted; (b) foam-assisted.

process, the residual gas saturation increases through foam trapping gas bubbles. The enlarged swept area provides higher capacity for trapping of $\mathrm{CO}_{2}$.

Note that in Fig. 7(b), the residual trapped $\mathrm{CO}_{2}$ saturation is a greater in the override zone where foam can not be present, which is caused by model assumptions. But this amount of trapped $\mathrm{CO}_{2}$ is not significant, and $\mathrm{CO}_{2}$ dissolves into brine gradually over time, as shown in Fig. 7(a). The residual $\mathrm{CO}_{2}$ is present mainly near the well, where foam is stable. In practice, one could increase the injection pressure to expand the swept area and move segregation point farther from the well.

Fig. 9 and Fig. 10 show the mole-fraction distribution of $\mathrm{CO}_{2}$ with time. $\mathrm{CO}_{2}$ fingers move downwards and grow gradually in both cases. The fingers between the override zone and bottom brine form earlier in brine-assisted $\mathrm{CO}_{2}$ injection because override happens rapidly (Fig. 9(a) and Fig. 10(a)). Finally, the average $\mathrm{CO}_{2}$ concentration in the whole domain (excluding the residual trapped region) in brine-assisted injection is higher than that with foam-injection. Once the tips of fingers reach the bottom boundary of the domain, $\mathrm{CO}_{2}$ fingers start to expand in the horizontal direction and merge with others. The number of fingers therefore is reduced, resulting from the mutual interaction between the fingers during the diffusion process. Note that the brine-assisted and foam-assisted $\mathrm{CO}_{2}$ injection shows similar behavior, including the migration and dissolution of the $\mathrm{CO}_{2}$ plume. The injection of foam is mainly applied to prevent $\mathrm{CO}_{2}$ from migrating upwards and reduce the breakthrough time during the injection period: the effects of foam on $\mathrm{CO}_{2}$ plume migration and dissolution at the upper layers at later time are negligible. In order to observe how the leading tip propagation changes with time, we show the results in foamassisted injection (Fig. 11) where the leading tip stops before it reaches the right boundary. As mentioned above, foam doesn't affect the migration of the $\mathrm{CO}_{2}$ override zone, so this result can represent the behavior of the $\mathrm{CO}_{2}$ plume for either brine-assisted or foam-assisted $\mathrm{CO}_{2}$ co-injection strategies in the post-injection period as long as the domain is long enough. The plume speed decreases with time until 

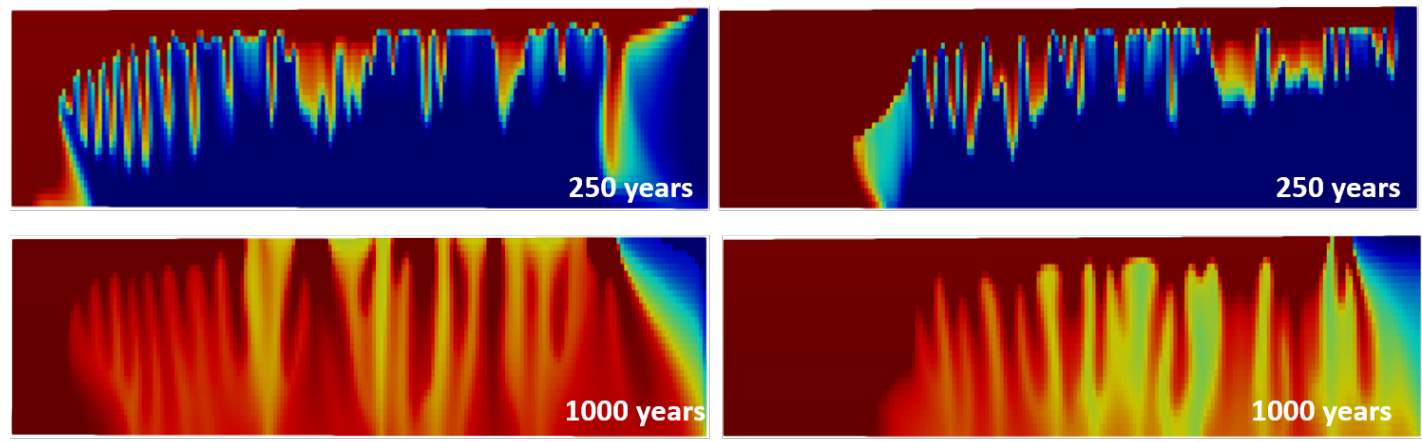

(a)

$6.8 \mathrm{e}-04$

$0.004 \quad 0.006 \quad 0.008$

$0.01 \quad 0.012 \quad 0.014$

(b)

$1.7 \mathrm{e}-02$

Figure 9: Concentration (mol/mol) profile of $\mathrm{CO}_{2}$ (front view) after 250 and 1000 years. (a) brineassisted; (b) foam-assisted.

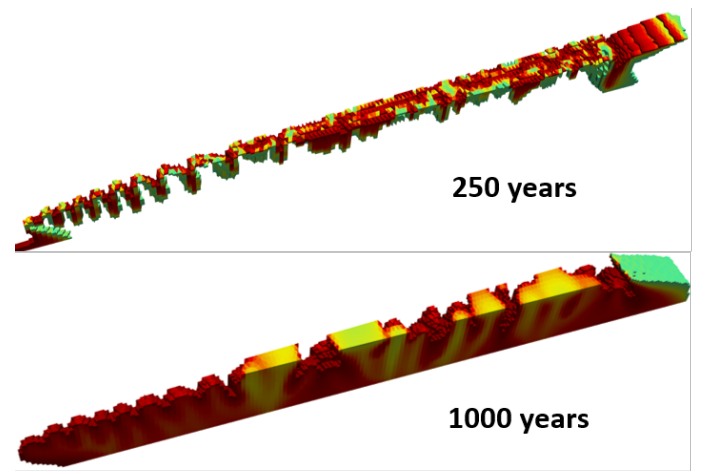

(a)
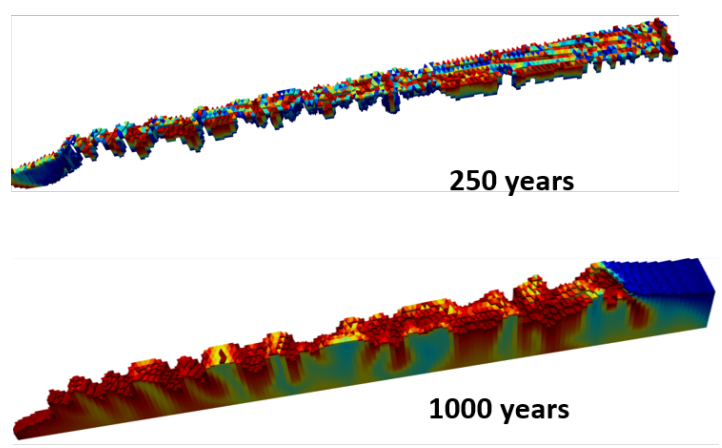

(b)

$6.8 e-04$

$0.004 \quad 0.006 \quad 0.008$

0.01

0.0120 .014

$1.7 e-02$

Figure 10: Concentration (mol/mol) profile of $\mathrm{CO}_{2}$ with threshold (3D) view after 250 and 1000 years. (a) brine-assisted; (b) foam-assisted. The concentration of threshold is $(0.005,0.016)$ in both cases.

the plume stops and retracts after about approximately 150 years: $370 \mathrm{~m}$ away from the injection point. The presence of the CTZ causes a reduction in leading tip speed. Our results show a similar trend to those of Elenius et al. (2015). This interaction between the speed of the leading tip and convective mixing also can be observed from the distribution of dissolved $\mathrm{CO}_{2}$ under the plume, see Fig. 8 .

Fig. 12a displays the global mass transfer into the single-phase brine region, which is defined as the amount of $\mathrm{CO}_{2}$ entering the single-phase region per unit time: $R=d M_{\mathrm{CO} 2} / d t$. Both injection strategies show similar results: $R$ increases at early time and later decreases with time. As shown in Elenius et al. (2015), the global mass-transfer decreases gradually after the $t_{\text {peel }}$, which is different from our simulation results. In our simulation, the thickness of the domain is just $30 \mathrm{~m}$, which causes the fingers reaching the bottom boundary in a very short time (around 150 years). Once the fingers arrive at the bottom, the dissolution rate starts to decrease, also seen in Fig. 3. With foam injection, $R$ increases faster at early time and reaches a slightly lower peak. On the one hand, once the injection ceases, foam sweeps much more area, increasing $\mathrm{CO}_{2}$ trapping, leading to a higher dissolution rate over a short period. On the other hand, the increased residual gas reduces the amount the $\mathrm{CO}_{2}$ which can dissolve into brine.

In this work, all properties are dependent on pressure, temperature and molar composition of each component. Therefore, Eq. 25 and Eq. 26, are not necessarily valid. However, in the post-injection process, the variation of pressure is slight ( $\sim 3$ bar), and we assume constant temperature. Therefore we still can use Eq. 25 to approximate the enhanced dissolution rate due to the presence of the CTZ. Note all 


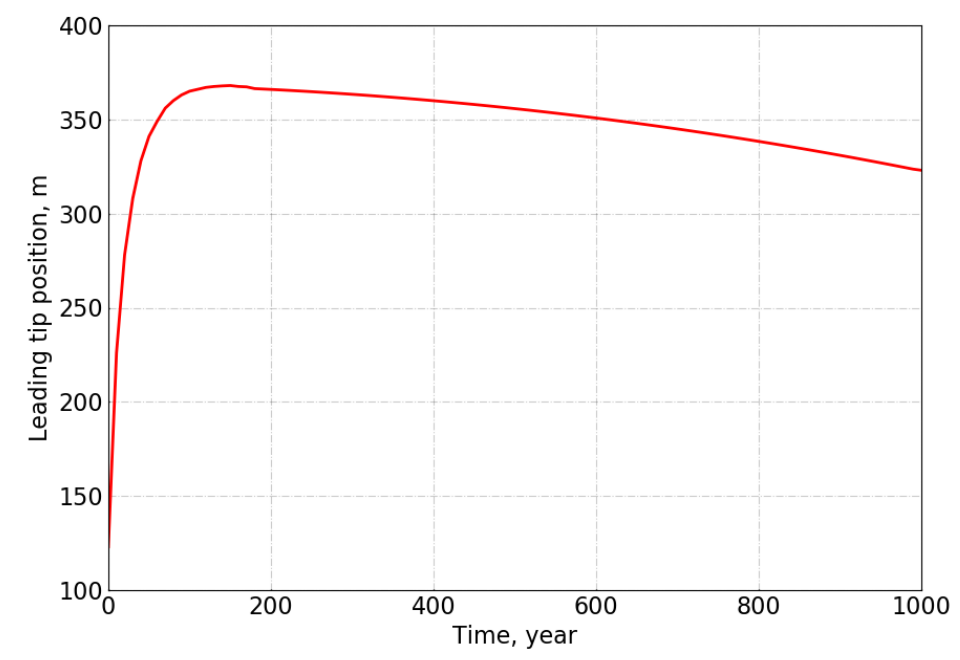

Figure 11: Position of the leading tip in foam-assisted injection process.

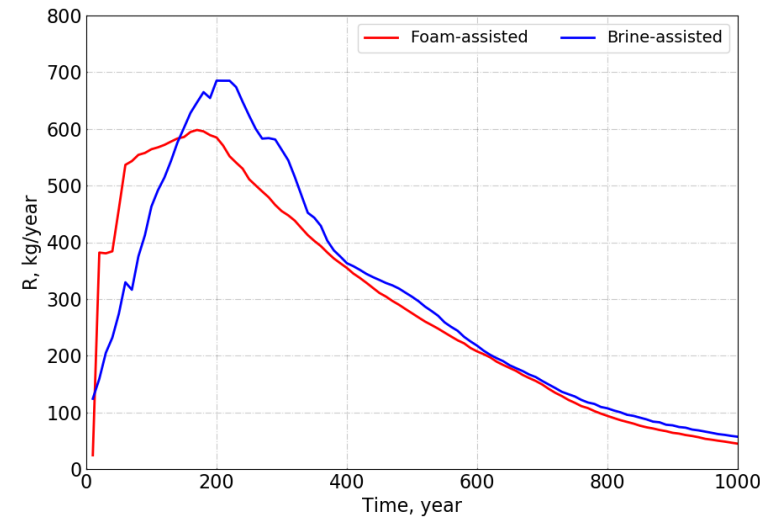

(a) Total mass transfer rate

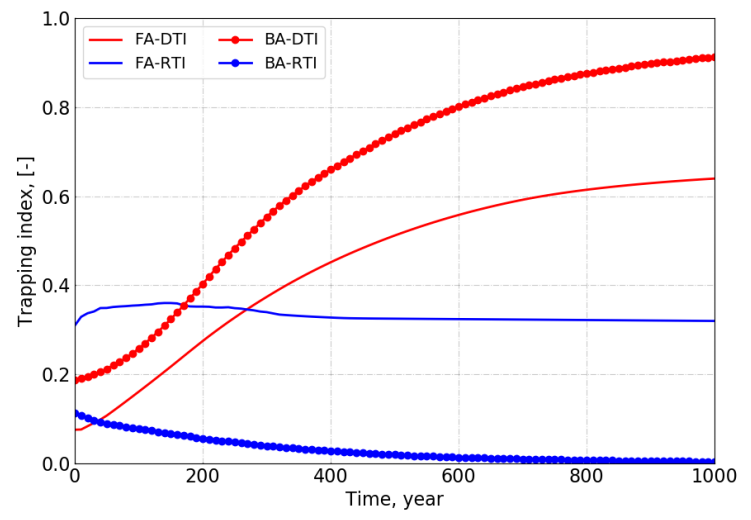

(b) Trapping index

Figure 12: (a) Total mass transfer rate $R$ of $\mathrm{CO}_{2}$ into the single-phase brine region; (b) Variation of trapping index in different mechanisms. FA: foam-assisted $\mathrm{CO}_{2}$ injection; $\mathrm{BA}$ : brine-assisted $\mathrm{CO}_{2}$ injection.

the properties in Eq. 25 are average: for instance, we calculate all water densities in all elements of the mesh and divide it by the total number of elements to get the corresponding water density. Here, $\Delta \rho_{w}$ $=5.75 \mathrm{~kg} / \mathrm{m}^{3}, X_{\max }=0.017 \mathrm{~mol} / \mathrm{mol}(0.0415 \mathrm{~kg} / \mathrm{kg}), \rho_{w}\left(X_{\max }\right)=982.6 \mathrm{~kg} / \mathrm{m}^{3}$, and $\mu_{w}=0.86 \mathrm{cp}$. We then obtain the average dissolution rate with the CTZ, $F_{\text {ave }}=0.254 \mathrm{~kg} /\left(\mathrm{m}^{3}\right.$ year) (Eq. 25). We compare this analytical dissolution rate with our simulation results. In brine-assisted $\mathrm{CO}_{2}$ injection, $R_{\max }=680$ $\mathrm{kg} /$ year, corresponding to $F_{\max }=0.325 \mathrm{~kg} /\left(\mathrm{m}^{3}\right.$ year $)\left(F_{\max }=R_{\max } /(A \times \phi)\right)$. This dissolution rate is $27.9 \%$ larger than that of analytical solution.

Considering the trapping mechanisms and time scale in this research, we estimate the effectiveness of $\mathrm{CO}_{2}$ geological storage, and three trapping indices are used to represent the contribution of residual trapping and dissolution trapping mechanism,

$$
\begin{aligned}
\text { Residual trapping index }(\mathrm{RTI}) & =\frac{\text { Total mass of residually trapped } \mathrm{CO}_{2}(\mathrm{~kg})}{\text { Total mass of injected } \mathrm{CO}_{2}(\mathrm{~kg})} \\
\text { Dissolution trapping index }(\mathrm{DTI}) & =\frac{\text { Total mass of dissolved } \mathrm{CO}_{2}(\mathrm{~kg})}{\text { Total mass of injected } \mathrm{CO}_{2}(\mathrm{~kg})}
\end{aligned}
$$

Fig. $12 \mathrm{~b}$ shows the variation of the trapping indices of different injection strategies over time. The $\mathrm{CO}_{2}$ plume moves further from the well and enlarges the contact area between the plume and formation brine after shutting off the well. Thus enables much more efficient dissolution of $\mathrm{CO}_{2}$ into the aqueous phase at the two-phase interface; DTI increases accordingly. The capacity for dissolving $\mathrm{CO}_{2}$ in brine-assisted 
$\mathrm{CO}_{2}$ injection is much greater while the amount of residually trapped $\mathrm{CO}_{2}$ is lower. The variation of RTI is opposite to that of DTI in both cases and less significant in brine-assisted $\mathrm{CO}_{2}$ injection. However, residual trapping plays a more important role in foam-assisted injection, with a greater trapping index (0.32). After 1000 years, around $92.5 \%$ of $\mathrm{CO}_{2}$ is dissolved into brine after co-injecting brine and $\mathrm{CO}_{2}$ compared to $62.3 \%$ of dissolved $\mathrm{CO}_{2}$ with foam-injection. In total, $94.3 \%$ of $\mathrm{CO}_{2}$ is trapped in foamassisted $\mathrm{CO}_{2}$ injection, increased by around $1.5 \%$ compared to brine-assisted $\mathrm{CO}_{2}$ injection.

As mentioned above, foam can mitigate gravity override during $\mathrm{CO}_{2}$ injection and reduce the risk of leakage or breakthrough. At early time, foam can improve the amount of trapped $\mathrm{CO}_{2}$, but in the long run, with the increasing ability of dissolution, the mechanism of residual trapping may play a lessimportant role. More-accurate modeling is required to predict the foam characteristics in $\mathrm{CO}_{2}$ storage processes.

\section{Conclusions}

In this work, we develop and validate a realistic full-physics phase-behavior model for simulation of $\mathrm{CO}_{2}$ sequestration in aquifers. The consistent thermodynamic model, based on a combination of a classic cubic equation of state (EOS) for gas components with an activity model for the aqueous phase, can accurately predict the complex phase behavior of the $\mathrm{CO}_{2}$ plume in brine. Comparison with the analytical solution in a simplified 2D setting verifies the capacity of DARTS implementation to predict the enhanced dissolution rate caused by the gravitational instabilities in the presence of a capillary transition zone (CTZ). An advanced numerical performance provided by the Operator-Based Linearization scheme allows us to perform full-physics simulation in a $3 \mathrm{D}$ sector model. The $\mathrm{CO}_{2}$ sequestration physics is complemented with a foam model which provides us the ability to investigate the effect of foam co-injection on $\mathrm{CO}_{2}$ trapping.

Foam injection can mitigate gravity override during gas injection by reducing gas mobility. This process increases the amount of residual trapped $\mathrm{CO}_{2}$ by $32.0 \%$ in this study. In addition, the presence of foam reduces the amount of flowing gas, thus reducing the risk of leakage. With a more realistic treatment of convective dissolution in 3D settings, the predicted average dissolution rate is almost $30 \%$ larger than that predicted by the analytical model. The presence of a capillary transition zone enhances dissolution rate, which is accurately captured in our model.

In this study, we use a simple foam model to investigate the effect of foam co-injection to $\mathrm{CO}_{2}$ trapping. This model does not capture all the characteristics, but it still represents some of the most important mechanisms of foam-assisted $\mathrm{CO}_{2}$ injection. For practical applications, foam generation and coalescence should be included into the physical model, and gas trapping should be represented more completely. There are other essential issues, such as the cost of surfactant, the depletion of surfactant over time, and the foam injectivity, to be considered. These factors will be taken into account in the future research.

\section{Acknowledgements}

We thank Mark Khait for his valuable contribution in DARTS implementation and Niek Morshuis for his contribution to the physics validation. We also acknowledge the financial support of China Scholarship Council.

\section{References}

Ajoma, E., Sungkachart, T., Ge, J., Le-Hussain, F. et al. [2020] Water-saturated CO2 injection to improve oil recovery and CO2 storage. Applied Energy, 266, 114853.

Alcorn, Z.P., Fredriksen, S.B., Sharma, M., Rognmo, A.U., Føyen, T.L., Fernø, M.A., Graue, A. et al. [2019] An Integrated Carbon-Dioxide-Foam Enhanced-Oil-Recovery Pilot Program With Combined Carbon Capture, Utilization, and Storage in an Onshore Texas Heterogeneous Carbonate Field. SPE Reservoir Evaluation \& Engineering.

Bachu, S., Bonijoly, D., Bradshaw, J., Burruss, R., Holloway, S., Christensen, N.P. and Mathiassen, O.M. [2007] CO2 storage capacity estimation: Methodology and gaps. International journal of greenhouse gas control, 1(4), 430-443. 
Bedrikovetsky, P. [2003] WAG displacements of oil-condensates accounting for hydrocarbon ganglia. Transport in porous media, 52(2), 229-266.

Bikerman, J.J. [1973] Foams, 10. Springer Science \& Business Media.

Caudle, B., Dyes, A. et al. [1958] Improving miscible displacement by gas-water injection. Transactions of the AIME, 213(01), 281-283.

Celia, M.A. and Nordbotten, J.M. [2009] Practical modeling approaches for geological storage of carbon dioxide. Groundwater, 47(5), 627-638.

CMG-STARS [2012] Computer Modeling Group Ltd. Calgary, AB, Canada.

Dholkawala, Z.F., Sarma, H. and Kam, S. [2007] Application of fractional flow theory to foams in porous media. Journal of Petroleum Science and Engineering, 57(1-2), 152-165.

Dong, X., Liu, H., Chen, Z., Wu, K., Lu, N. and Zhang, Q. [2019] Enhanced oil recovery techniques for heavy oil and oilsands reservoirs after steam injection. Applied energy, 239, 1190-1211.

Elenius, M., Voskov, D. and Tchelepi, H. [2015] Interactions between gravity currents and convective dissolution. Advances in Water Resources, 83, 77-88.

Elenius, M.T., Nordbotten, J.M. and Kalisch, H. [2012] Effects of a capillary transition zone on the stability of a diffusive boundary layer. The IMA Journal of Applied Mathematics, 77(6), 771-787.

Elenius, M.T., Nordbotten, J.M. and Kalisch, H. [2014] Convective mixing influenced by the capillary transition zone. Computational Geosciences, 18(3-4), 417-431.

Friedmann, F., Chen, W., Gauglitz, P. et al. [1991] Experimental and simulation study of hightemperature foam displacement in porous media. SPE reservoir engineering, 6(01), 37-45.

Gale, J. [2004] Geological storage of $\mathrm{CO} 2$ : What do we know, where are the gaps and what more needs to be done? Energy, 29(9-10), 1329-1338.

Hesse, M.A., Orr, F.M. and Tchelepi, H. [2008] Gravity currents with residual trapping. Journal of Fluid Mechanics, 611, 35-60.

IPCC, I.P.O.C. [2014] Climate Change 2013: The physical science basis: Working group I contribution to the fifth assessment report of the Intergovernmental Panel on Climate Change. Cambridge University Press.

Iranshahr, A., Voskov, D. and Tchelepi, H. [2010] Generalized negative-flash method for multiphase multicomponent systems. Fluid Phase Equilibria, 299(2), 272-284.

Izadi, M. and Kam, S. [2018] Investigating Supercritical CO 2 Foam Propagation Distance: Conversion From Strong Foam to Weak Foam vs. Gravity Segregation. Transport in Porous Media, 1-28.

Jones, S., Getrouw, N. and Vincent-Bonnieu, S. [2018] Foam flow in a model porous medium: II. The effect of trapped gas. Soft matter, 14(18), 3497-3503.

Kala, K. and Voskov, D. [2020] Element balance formulation in reactive compositional flow and transport with parameterization technique. Computational Geosciences, 24(2), 609-624.

Khait, M. and Voskov, D. [2017] Operator-based linearization for general purpose reservoir simulation. Journal of Petroleum Science and Engineering, 157, 990-998.

Khait, M. and Voskov, D. [2018] Adaptive parameterization for solving of thermal/compositional nonlinear flow and transport with buoyancy. SPE Journal, 23, 522-534.

Kil, R.A., Nguyen, Q.P., Rossen, W.R. et al. [2011] Determining trapped gas in foam from computedtomography images. SPE Journal, 16(01), 24-34.

Kritchevsky, I. and Iliinskaya, A. [1945] Partial molal volumes of gases dissolved in liquids.(A contribution to the thermodynamics of dilute solutions of non-electrolytes). Acta Physicochim. URSS, 20(3), 327-348.

Li, D., Zhang, H., Li, Y., Xu, W. and Jiang, X. [2018] Effects of N2 and H2S binary impurities on CO2 geological storage in stratified formation-A sensitivity study. Applied Energy, 229, 482-492.

Lotfollahi, M., Farajzadeh, R., Delshad, M., Varavei, A. and Rossen, W.R. [2016] Comparison of implicit-texture and population-balance foam models. Journal of Natural Gas Science and Engineering, 31, 184-197.

Lyu, X., Liu, H., Pang, Z. and Sun, Z. [2018] Visualized study of thermochemistry assisted steam flooding to improve oil recovery in heavy oil reservoir with glass micromodels. Fuel, 218, 118-126.

Morshuis, N. [2019] An improved carbon dioxide thermodynamic model applied for reservoir simulation: Implementation of an improved thermodynamic model in Delft Advanced Terra Simulator (DARTS) and an investigation into the effects of impurities on gas plume behaviour.

NASA [2018] Long-Term Warming Trend Continued in 2017: NASA, NOAA. 
Pruess, K., García, J., Kovscek, T., Oldenburg, C., Rutqvist, J., Steefel, C. and Xu, T. [2004] Code intercomparison builds confidence in numerical simulation models for geologic disposal of $\mathrm{CO} 2$. Energy, 29(9-10), 1431-1444.

Qi, R., LaForce, T.C. and Blunt, M.J. [2009] Design of carbon dioxide storage in aquifers. International Journal of Greenhouse Gas Control, 3(2), 195-205.

Raziperchikolaee, S., Alvarado, V. and Yin, S. [2013] Effect of hydraulic fracturing on long-term storage of $\mathrm{CO} 2$ in stimulated saline aquifers. Applied energy, 102, 1091-1104.

Rossen, W.R. [1996] Foams in enhanced oil recovery. Foams: Theory, Measurements and Applications, 57, 413-464.

Rossen, W.R., Van Duijn, C., Nguyen, Q.P., Shen, C., Vikingstad, A.K. et al. [2010] Injection strategies to overcome gravity segregation in simultaneous gas and water injection into homogeneous reservoirs. SPE Journal, 15(01), 76-90.

Schramm, L.L. [1994] Foams: fundamentals and applications in the petroleum industry. ACS Publications.

Slim, A.C. [2014] Solutal-convection regimes in a two-dimensional porous medium. Journal of fluid mechanics, 741, 461-491.

Spycher, N., Pruess, K. and Ennis-King, J. [2003] CO2-H2O mixtures in the geological sequestration of CO2. I. Assessment and calculation of mutual solubilities from 12 to $100 \mathrm{C}$ and up to 600 bar. Geochimica et cosmochimica acta, 67(16), 3015-3031.

Stone, H.L. et al. [1982] Vertical, conformance in an alternating water-miscible gas flood. In: SPE annual technical conference and exhibition. Society of Petroleum Engineers.

Tang, G.Q. and Kovscek, A. [2006] Trapped gas fraction during steady-state foam flow. Transport in porous media, $\mathbf{6 5}(2), 287-307$.

Vitoonkijvanich, S., AlSofi, A.M. and Blunt, M.J. [2015] Design of foam-assisted carbon dioxide storage in a North Sea aquifer using streamline-based simulation. International Journal of Greenhouse Gas Control, 33, 113-121.

Voskov, D. [2017] Operator-based linearization approach for modeling of multiphase multi-component flow in porous media. Journal of Computational Physics, 337, 275-288.

Wang, Y., Voskov, D., Khait, M. and Bruhn, D. [2020] An efficient numerical simulator for geothermal simulation: A benchmark study. Applied Energy, 264.

Ziabakhsh-Ganji, Z. and Kooi, H. [2012] An Equation of State for thermodynamic equilibrium of gas mixtures and brines to allow simulation of the effects of impurities in subsurface $\mathrm{CO} 2$ storage. International Journal of Greenhouse Gas Control, 11, S21-S34. 